\title{
$\mathrm{BMJ}$
}

\section{Continued cannabis use and risk of incidence and persistence of psychotic symptoms: 10 year follow-up cohort study}

\begin{abstract}
Rebecca Kuepper, research psychologist, ${ }^{1}$ Jim van Os, professor, ${ }^{1}$ visiting professor, ${ }^{2}$ Roselind Lieb, professor, ${ }^{3,4}$ Hans-Ulrich Wittchen, professor, ${ }^{4,5}$ Michael Höfler, research statistician, ${ }^{5}$ Cécile Henquet, lecturer $^{1}$
\end{abstract}

${ }^{1}$ Department of Psychiatry and Neuropsychology, South Limburg Mental Health Research and Teaching Network, EURON, Maastricht University Medical Center, Maastricht, Netherlands

2King's College London, King's Health Partners, Department of

Psychosis Studies, Institute of Psychiatry, London, UK

${ }^{3}$ Department of Psychology,

Division of Epidemiology and

Health Psychology, University of

Basel, Switzerland

${ }^{4}$ Max Planck Institute of Psychiatry, Munich, Germany ${ }^{5}$ Institute of Clinical Psychology and Psychotherapy, Technical University Dresden, Germany

Correspondence to: J van Os, Department of Psychiatry and Neuropsychology, South Limburg Mental Health Research and

Teaching Network, EURON,

Maastricht University Medical

Center, PO Box 616, NL-6200 MD,

Maastricht, Netherlands

j.vanos@sp.unimaas.nl

Cite this as: $B M J$ J 2011;342:d738 doi:10.1136/bmi.d738

\section{ABSTRACT}

Objective To determine whether use of cannabis in adolescence increases the risk for psychotic outcomes by affecting the incidence and persistence of subclinical expression of psychosis in the general population (that is, expression of psychosis below the level required for a clinical diagnosis).

Design Analysis of data from a prospective population based cohort study in Germany (early developmental stages of psychopathology study).

Setting Population based cohort study in Germany. Participants 1923 individuals from the general population, aged 14-24 at baseline.

Main outcome measure Incidence and persistence of subthreshold psychotic symptoms after use of cannabis in adolescence. Cannabis use and psychotic symptoms were assessed at three time points (baseline, T2 (3.5 years), T3 (8.4 years)) over a 10 year follow-up period with the Munich version of the composite international diagnostic interview (M-CIDI).

Results In individuals who had no reported lifetime psychotic symptoms and no reported lifetime cannabis use at baseline, incident cannabis use over the period from baseline to $\mathrm{T} 2$ increased the risk of later incident psychotic symptoms over the period from T2 to T3 (adjusted odds ratio 1.9, 95\% confidence interval 1.1 to $3.1 ; \mathrm{P}=0.021$ ). Furthermore, continued use of cannabis increased the risk of persistent psychotic symptoms over the period from T2 to T3 $(2.2,1.2$ to 4.2; $\mathrm{P}=0.016)$. The incidence rate of psychotic symptoms over the period from baseline to T2 was 31\% (152) in exposed individuals versus $20 \%$ (284) in non-exposed individuals; over the period from T2 to T3 these rates were 14\% (108) and $8 \%$ (49), respectively.

Conclusion Cannabis use is a risk factor for the development of incident psychotic symptoms. Continued cannabis use might increase the risk for psychotic disorder by impacting on the persistence of symptoms.

\section{INTRODUCTION}

Cannabis is the most commonly used illicit drug in the world, particularly among adolescents. ${ }^{12}$ The use of cannabis is consistently associated with mental illness, ${ }^{3}$ in particular psychotic disorder. ${ }^{4-9}$ It remains a matter of debate, however, whether the association between cannabis and psychosis is causal, or whether early psychotic experiences might in fact prompt cannabis use as a means of self medication. ${ }^{1011}$ This issue can be resolved only if incident cannabis use is investigated in relation to later incident psychotic symptoms or disorder. Rarely have studies been able to examine the longitudinal relation between cannabis use and psychosis in this fashion.

The issue of self medication was addressed by Henquet and colleagues, ${ }^{6}$ using data from the German prospective early developmental stages of psychopathology study. ${ }^{12} 13$ The authors investigated the association between cannabis use at baseline and subsequent development of psychotic symptoms at four year follow-up and reported that after adjustment for pre-existing psychotic symptoms, cannabis use at baseline still remained significantly associated with psychotic symptoms at follow-up. There was no evidence of an effect of self medication as pre-existing psychotic symptoms did not significantly predict later cannabis use. ${ }^{6}$ Ferdinand and co-workers investigated the role of pre-existing self reported psychotic symptoms and showed a bi-directional association between cannabis and psychotic symptoms over a 14 year follow-up study in the general population. ${ }^{11}$ They showed that cannabis use predicted later psychotic symptoms in individuals with no evidence of psychotic symptoms before starting to use cannabis and that the reverse was also true, in that psychotic symptoms predicted cannabis use in those who had not used cannabis before the onset of those symptoms. ${ }^{11}$ A prospective population based cohort study also found evidence for a self medication effect. ${ }^{14}$ Individuals with self reported hallucinations at the age of 14 had a higher risk of using cannabis on a daily basis at the age of 21 . In a sibling pair analysis, however, this study also suggested an independent effect of cannabis use on self reported delusional ideation later in life. ${ }^{14}$ Thus, although the cannabis-psychosis link has been investigated in 
many studies, results on the temporal association between cannabis use and psychotic symptoms remain conflicting. Longitudinal cohort studies with multiple repeated interview based measures of cannabis use and psychotic symptoms are needed to clarify this issue. The EDSP study, ${ }^{1213}$ which completed its recent 10 year follow-up representing the fourth assessment (assessments at baseline, T1, T2, and T3, see also fig 1), is uniquely suitable for the investigation of the temporal association between cannabis and psychosis.

Another issue is the mechanism by which cannabis might increase the risk of psychotic symptoms, particularly whether it might increase the risk by causing persistence of normally transitory developmental expression of psychotic experiences. For most individuals, subclinical expression of psychotic phenomena (that is, expression of psychosis below the level required for a clinical diagnosis) is transitory and never progresses to psychotic illness. ${ }^{15}$ Subthreshold psychotic experiences could, however, become abnormally persistent, depending on the degree of additional exposure to environmental risk factors, ${ }^{16-18}$ and progressively greater levels of persistence might be associated with a greater risk for transition to clinical psychotic disorder. ${ }^{19}$ Spauwen and colleagues showed that the persistence rate of psychotic experiences was much higher for individuals growing up in an urban rather than a rural environment. ${ }^{16}$ Similarly, Cougnard and co-workers provided evidence that childhood trauma, urban environment, and cannabis act additively in increasing the risk of persistence of psychotic experiences. ${ }^{17}$ The fact that cannabis use increases risk of psychosis in a dose-response fashion ${ }^{61420}$ and that patients with psychosis who continue to use cannabis show more severe and persistent symptoms ${ }^{21}$ suggests that cannabis use might increase the risk for psychotic illness by impacting on the persistence rate of psychotic experiences that under normal circumstances (that is, without exposure to cannabis) would have remained transitory phenomena for most people. In a population based 10 year follow-up cohort study of adolescents and young adults, we investigated the association

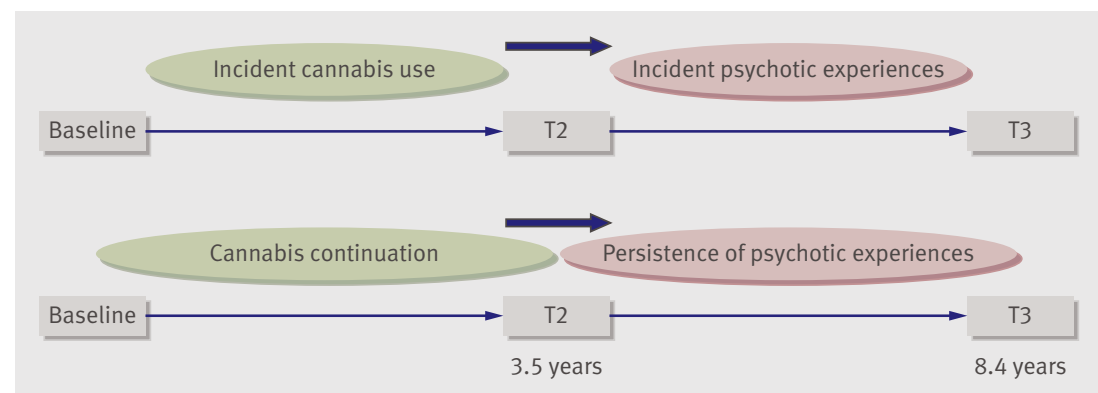

Fig 1 Study design. Top: testing association between incident cannabis use with onset in period from baseline to T2 and incident psychotic symptoms with onset in period from T2 to T3 in individuals who had not used cannabis at baseline and who had not reported any psychotic experience at $\mathrm{T} 2$ (that is, no lifetime psychotic experiences by $\mathrm{T} 2$ ). Bottom: testing association between different cannabis exposure states (combinations of cannabis use at baseline (lifetime), or T2 or both (interval) and persistence of psychotic experiences (that is, presence of psychotic experiences at both T2 (lifetime) and T3 (interval))
Table 1|Characteristics of participants by use of cannabis at T2 (3.5 years after baseline) $(n=1923)$. Figures are numbers (percentages)

\begin{tabular}{|c|c|c|}
\hline & $\begin{array}{l}\text { Used cannabis } \\
\qquad(n=393)\end{array}$ & $\begin{array}{l}\text { Did not use cannabis } \\
\qquad(\mathrm{n}=1530)\end{array}$ \\
\hline Men & $119(30)$ & $807(53)$ \\
\hline Women & $274(70)$ & $723(47)$ \\
\hline \multicolumn{3}{|c|}{ Socioeconomic status*: } \\
\hline Lower & $30(7)$ & $85(6)$ \\
\hline Middle & $220(54)$ & 894 (59) \\
\hline Upper & $137(34)$ & $524(35)$ \\
\hline Other & $22(5)$ & $6(0.4)$ \\
\hline \multicolumn{3}{|c|}{ Urban/rural environment†: } \\
\hline Urban & $295(75)$ & $1050(69)$ \\
\hline Rural & $98(25)$ & $480(31)$ \\
\hline \multicolumn{3}{|c|}{ Childhood traumał: } \\
\hline Yes & $97(25)$ & $266(17)$ \\
\hline No & $296(75)$ & $1264(83)$ \\
\hline
\end{tabular}

Use of other drugs at baseline§:

\begin{tabular}{|c|c|c|}
\hline Yes & $25(6)$ & $11(1)$ \\
\hline No & $368(94)$ & $1519(99)$ \\
\hline \multicolumn{3}{|c|}{ Use of other drugs at T2§: } \\
\hline Yes & $40(10)$ & $3(0.2)$ \\
\hline No & $353(90)$ & $1527(99)$ \\
\hline \multicolumn{3}{|c|}{ Any psychiatric disorder at baseline ${ }^{\star \star ף}$ : } \\
\hline Yes & $76(19)$ & $180(12)$ \\
\hline No & $317(81)$ & $1350(88)$ \\
\hline
\end{tabular}

*Socioeconomic status: lower (lower class, lower middle class), middle (middle middle class), upper (higher middle class, upper class), other (none of the above or missing). Data missing for five participants. †Urban (city of Munich, $10559 / \mathrm{km}^{2}$ ), rural (surroundings of Munich, $1432 / \mathrm{km}^{2}$ ).

$\ddagger$ Childhood trauma: any traumatic experience during childhood.

§On more than five occasions.

TOther than psychosis, according to M-CIDI diagnoses.

between incident cannabis use and true incidence of psychotic experiences (that is, after exclusion of individuals with lifetime pre-existing psychotic experiences) and risk of persistence of psychotic experiences.

\section{METHOD}

\section{Sample and study design}

The observation frame was part of the early developmental stages of psychopathology (EDSP) study, which collected data on the prevalence, incidence, risk factors, comorbidity, and course of mental disorders in a random representative population sample of adolescents and young adults in the general population. ${ }^{1213}$ After ethical approval, the baseline sample was randomly drawn in 1994 from the respective population registry offices of Munich and its 29 counties to mirror the distribution of individuals aged 14-24 at the time of the baseline interview in 1995. The base population comprised people born from 1 June 1970 to 31 May 1981 registered as residents in these localities and having German citizenship. These registers are highly accurate because each German is registered by his or her town, registers are regularly updated, for scientific studies any number of randomly drawn addresses with a given sex and age group can be 


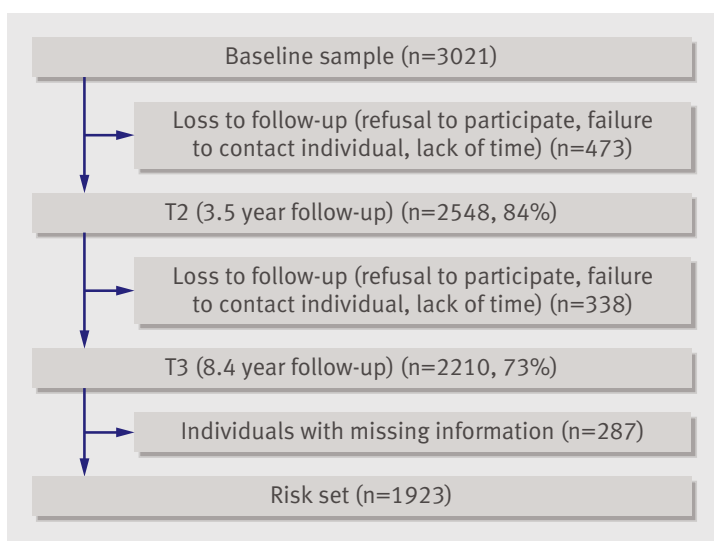

Fig 2 Response rates and reasons for loss to follow-up

obtained, and registration is strictly enforced by law and the police. More details on the sampling, representativeness, instruments, procedures, and statistical methods of the study sample can be found elsewhere. ${ }^{1213}$

The overall design of study is longitudinal and prospective, consisting of a baseline and three follow-up surveys. The first (baseline to T1) covered a mean of 1 . 6 years (SD 0.2), the second (baseline to T2) covered a mean of 3.5 years (SD 0.3), and the third (baseline to T3) covered a mean of 8.4 years (range 7.3-10.5, SD 0.7). Because our primary goal was to examine the incidence and developmental risk factors for psychopathology, we sampled the younger group (age 14$15)$, presumed to have the highest incidence density, at twice the rate of people aged 16-21 and sampled the oldest group (age 22-24) at half this rate. For the same reason we examined people aged 14-17 at baseline at the four time points and those aged 18-24 only three times (baseline, T2, T3). The present study is based on the whole cohort assessed at baseline, T2, and T3. Figure 1 shows a schematic illustration of the overall design and the current analyses. Response rates were $84 \%(2548)$ at $\mathrm{T} 2$ and $73 \%(2210)$ at T3 (fig 2).

\section{Instruments}

We used the computerised version of the Munich composite international diagnostic interview (DIA-X/M-

Table 2 | Patterns of cannabis use in relation to presence of psychotic symptoms* at T2 ( 3. 5 years after baseline) and T3 (8.4 years after baseline) in risk set $(n=1923)$. Figures are numbers (percentage†) of participants

\begin{tabular}{lccccc} 
& \multicolumn{2}{c}{ Psychotic symptoms at T2 } & & \multicolumn{2}{c}{ Psychotic symptoms at T3 } \\
\cline { 2 - 3 } \cline { 5 - 5 } Cannabis use $\begin{array}{l}\text { Yes } \\
\text { Baseline }\end{array}$ & \multicolumn{1}{c}{ Yes } & & Yes & No \\
\hline Yes & $81(4)$ & $166(9)$ & & $42(2)$ & $205(11)$ \\
\hline No & $355(18)$ & $1321(69)$ & & $189(10)$ & $1487(77)$ \\
\hline T2 & $126(7)$ & $267(14)$ & & $69(4)$ & $324(17)$ \\
\hline Yes & $310(16)$ & $1220(64)$ & & $162(8)$ & $1368(71)$ \\
\hline No & & & & & \\
\hline
\end{tabular}

*Any psychotic symptom lifetime (T2) and interval (T3) as assessed with M-CIDI (G) section.

†Some percentages do not total 100 because of rounding.

¥On more than five occasions as assessed with M-CIDI (L) section.
CIDI) ${ }^{22}$ an updated version of the World Health Organization's CIDI version 1.2. ${ }^{23}$ The DIA-X/M-CIDI is a comprehensive fully standardised diagnostic interview that assesses symptoms, syndromes, and diagnoses of various mental disorders in accordance with the definitions and criteria of ICD-10 (international classification of diseases, 10th revision) and DSM-IV (Diagnostic and Statistical Manual of Mental Disorders, fourth edition), along with information about onset, duration, severity of symptoms, and psychosocial impairment. The CIDI has been primarily designed for use in epidemiological studies of mental disorders and can also be used for clinical purposes. It is divided into 16 sections: one sociodemographic section, 12 sections assessing 288 symptoms of groups of mental disorders (including somatoform and dissociative, phobic and other anxiety, depressive and dysthymic, manic and bipolar affective, schizophrenia and other psychotic, eating, dementia and other cognitive, post-traumatic stress, as well as tobacco, alcohol, and substance related disorders), and three final sections containing concluding questions, interviewers' observations, and interviewers' ratings. The instrument, designed for use by trained interviewers who are not clinicians, has shown high inter-rater ${ }^{2425}$ and test-retest reliability. ${ }^{226}$ The assessment of psychosis with CIDI by lay interviewers is not considered reliable so trained clinical interviewers at the level of clinical psychologist, who were allowed to probe with follow-up clinical questions, conducted the interviews in the respondents' homes. At baseline, the DIA-X/M-CIDI lifetime version was used. At each of the follow-up assessments, the interval version was used to assess the period from the last interview until the next. Data on the $\mathrm{G}$ section concerning psychosis and its clinical relevance were collected only at T2 (lifetime version) and T3 (interval version). As the assessment of substance use was part of the diagnostic interview with the DIA-X/M-CIDI, psychologists who did the interviews were not blinded for cannabis use.

\section{Assessment of psychotic symptoms}

Data on psychotic experiences were collected at time T2 (lifetime version) and T3 (interval version) with the $\mathrm{G}$ section of the DIA-X/M-CIDI. As the primary objective of the EDSP study was to investigate the early stages of substance misuse in adolescents and young adults, data on the occurrence of psychotic symptoms as assessed with the $G$ section were added at T2 (measuring lifetime experience of psychotic symptoms) and T3 (measuring interval experience of psychotic symptoms). ${ }^{13}$ As in previous work ${ }^{619}$ presence of psychotic experiences was broadly defined as any rating of present on any of the 20 DIAX/MCIDI core psychosis items (G1, G2a, G3-G5, G7G13, G13b, G14, G17, G18, G20, G20C, G21, and G22a), including 14 delusion items, five hallucination items, and one item on passivity phenomena. Items relate to classic psychotic symptoms involving, for example, persecution, thought interference, auditory hallucinations, and passivity phenomena. The 
Table 3| Association between incident cannabis use at T2 (3. 5 years after baseline) and incident psychotic experiences at T3 (8.4 years after baseline) Figures are odds ratios (95\% confidence intervals) and $P$ values

\begin{tabular}{lcc}
\multirow{2}{*}{\begin{tabular}{l} 
Cannabis use at T2 \\
\cline { 2 - 3 } Whole sample
\end{tabular}} & \multicolumn{2}{c}{ Risk of psychotic experiences at T3 } \\
\hline After exclusion $\dagger$ & $1.8(1.3$ to 2.4$),<0.001$ & $1.5(1.1$ to 2.1$), 0.018$ \\
\hline & $2.1(1.3$ to 3.4$), 0.004$ & $1.9(1.1$ to 3.1$), 0.021$
\end{tabular}

*Adjusted for age, sex, socioeconomic status, use of other drugs, childhood trauma, and urban/rural environment.

†Excludes individuals with baseline cannabis use and pre-existing psychotic symptoms.

psychologist invited participants to read a list of all the psychotic experiences and then asked them whether they ever experienced such symptoms (list and phrasing available on request). All these psychosis items were rated as absent or present. Thus, all the psychosis DIA-X/M-CIDI items used in the present study were coded in a dichotomous manner without intermediate levels. Psychotic symptoms at T2 accordingly referred to lifetime occurrence of at least one positive rating on any psychosis item in the $\mathrm{G}$ section; T3 psychotic symptoms denote interval occurrence of at least one positive rating on any psychosis item in this section.

\section{Assessment of cannabis use}

Cannabis use was assessed with the L-section of the DIA-X/M-CIDI at all three assessments. The question "Have you ever used cannabis five times or more?" was used to define cannabis exposure. As with previous work in this sample,${ }^{6}$ the CIDI cut off of use of five times or more was used to define cannabis exposure (yes versus no). Thus, at baseline, cannabis use was defined as lifetime use of cannabis of five times or more ("baseline cannabis use," dichotomously defined yes versus no). At T2, and similarly at T3, cannabis use was defined as interval use of cannabis of five times or more, during the intervals of baseline toT2 and $\mathrm{T} 2$ to $\mathrm{T} 3$, respectively (T2 cannabis use, T3 cannabis use, both dichotomously defined yes versus no). As assessment of frequency of cannabis use differed between time points (for instance, at baseline: How often do you use cannabis on a five point scale ranging from less than monthly to almost daily?; at T2: How many times have you used cannabis since the last assessment?), the frequency variable was not included in the analyses.

\section{Statistical analyses}

We used Stata, release 10.0 (StataCorp, College Station, TX) for data analysis and expressed associations as odds ratios from logistic regression models.

All analyses were a priori adjusted for age at baseline (in years), sex ( $0=$ female, $1=$ male), socioeconomic status (lower, middle, upper, other, as assessed at baseline), use of other drugs at baseline and T2, childhood trauma (that is, trauma before the age of 14 as assessed at baseline), ${ }^{27}$ and urban/rural environment. ${ }^{2829}$ The variable use of other drugs included psychostimulants, sedatives, opiates, cocaine, phencyclidine, and psychedelic drugs and refers to CIDI rated use of five times or more (lifetime or interval use at baseline and T2 respectively) of any of these drugs, as assessed with the L section of the M-CIDI. Childhood trauma refers to experience of any traumatic experience during childhood as assessed with the $\mathrm{N}$ section of the $\mathrm{M}$ CIDI at baseline and was dichotomously defined as present or absent. Fisher and colleagues have shown that self report questionnaires constitute a valid method of assessing childhood trauma in individuals presenting with psychotic features. ${ }^{30}$ Urban/rural environment was assessed at baseline by retrieving data on place of residence from the population registry offices of the city and the 29 counties of Munich and was defined as either living in the city of Munich (10 559 people per $\mathrm{km}^{2}$ ) or in the rural surroundings (1432 people per $\mathrm{km}^{2}$ ) at baseline.

To examine whether associations between cannabis use and psychotic symptoms were independent of other psychiatric diagnoses at baseline, we additionally adjusted analyses for any psychiatric diagnosis other than psychosis at baseline. We used a dichotomous variable of any psychiatric diagnosis (present versus absent), summarising main psychiatric diagnoses other than psychosis at baseline (such as major depressive disorder, hypomania, dysthymic disorder, bipolar disorder, panic disorder, obsessive compulsive disorder, post-traumatic stress disorder, conversion disorder, somatisation disorder, eating disorders).

We carried out sensitivity analyses to investigate whether attrition occurred at random and to assess potential bias introduced by missing data. This was done by multiple imputation of missing values with the ICE routine in Stata 11.1. This method imputes several alternative versions of the complete dataset from the available data.

\section{Incident use of cannabis and incidence of psychotic symptoms}

We used logistic regression analyses to investigate the association between incident cannabis use from baseline to $\mathrm{T} 2$ and incident psychosis outcome from $\mathrm{T} 2$ to T3 (see fig 1, top). To investigate the true incidence of psychotic symptoms after cannabis use, rather than persistence of pre-existing psychotic experiences, we excluded from the analysis individuals who had admitted to lifetime presence of any psychotic symptom at T2 $(\mathrm{n}=574,23 \%)$. We also excluded all individuals with cannabis use at baseline, thus including only individuals with new cannabis exposure between baseline and $\mathrm{T} 2$.

We investigated reverse causality (that is, self medication) by testing the association between psychosis at baseline-T2 and cannabis use at T2-T3 in those who had not used cannabis at both baseline and $\mathrm{T} 2$.

Cannabis use and risk of persistent psychotic symptoms We calculated a dichotomous persistence variable (no persistence versus persistence), with no persistence referring to experience of psychotic symptoms either never or only once (at T2 or T3) and persistence 
Table $4 \mid$ Course of psychotic experiences in relation to level of continued cannabis use at T2 (3.5 years after baseline) and T3 (8.4 years after baseline). Figures are numbers (percentages) of participants

\begin{tabular}{lccc}
\multirow{2}{*}{ Cannabis continuation } & \multicolumn{3}{c}{ Psychotic experiences at follow-up } \\
\cline { 2 - 4 } No use & None & At T2 or T3 & At T2 and T3 \\
\hline At baseline but not at T2 & $1071(75)$ & $303(21)$ & $64(4)$ \\
\hline At T2 but not at baseline & $144(60)$ & $25(27)$ & $8(9)$ \\
\hline At baseline and T2 & $90(58)$ & $45(32)$ & $19(8)$ \\
\hline
\end{tabular}

referring to experience of psychotic symptoms at both T2 and T3. To investigate whether different levels of exposure to cannabis affected persistence of psychotic symptoms differentially, we calculated a categorical cannabis continuation variable $(0=$ never used cannabis; $1=$ cannabis use at baseline but not at T2; $2=$ cannabis use at $\mathrm{T} 2$ but not at baseline, $3=$ cannabis use at both baseline and T2). We used logistic regression analyses to investigate the association between cannabis continuation and psychosis persistence (fig 1 bottom).

\section{RESULTS}

\section{Sample}

A total of 2210 individuals completed the T3 assessment; information on substance use and psychotic symptoms was missing for 287 participants, resulting in a final risk set for analysis of 1923 individuals, of whom 926 (48\%) were men (fig 2). Mean age was 18.3 (SD 3.3) at baseline, 21.8 (SD 3.4) at T2, and 26.6 (SD 3.5) at T3. Table 1 gives further characteristics of participants.

At baseline, 247 participants reported using cannabis (13\% lifetime use). Of those, 56 participants $(23 \%)$ used cannabis almost every day, $69(28 \%)$ reported weekly use, 57 (23\%) used cannabis monthly, and 65 $(26 \%)$ reported using cannabis less than once a month. At T2, 392 participants reported using cannabis (20\% interval use). The mean frequency of those who reported cannabis use at $\mathrm{T} 2$ was 130 times within the period from baseline to T2 (range 5-997). Psychotic symptoms were reported by 436 participants $(23 \%)$ at T2 (lifetime) and by 231 participants (12\%) at T3 (interval). Table 2 summarises patterns of cannabis use in relation to psychotic symptoms.

\section{Incident cannabis use and incidence of psychotic symptoms}

The incidence rate of psychotic symptoms over the period from baseline to T2 was 31\% (152) in exposed individuals and 20\% (284) in non-exposed individuals; over the period from T2 to T3 these rates were $14 \%$ (108) and 8\% (49), respectively.

Lifetime cannabis use as assessed at T2 significantly increased the risk of psychotic experiences at T3 (adjusted odds ratio 1.5, 95\% confidence interval 1.1 to $2.1 ; \mathrm{P}=0.018$; tables 3 and 4 ). After we excluded all individuals who had used cannabis at baseline and had reported psychotic experiences at T2, incident cannabis use over the period from baseline to T2 similarly increased the risk for incident psychotic experiences between $\mathrm{T} 2$ and $\mathrm{T} 3(1.9,1.1$ to $3.1, \mathrm{P}=0.021)$. Results were unchanged after additional adjustment for any psychiatric diagnosis other than psychosis at baseline $(1.9,1.1$ to $3.1 ; \mathrm{P}=0.020)$.

There was no evidence for self medication effects, as psychotic experiences at T2 did not predict incident cannabis use between $\mathrm{T} 2$ and $\mathrm{T} 3(0.8,0.6$ to 1.2 ; $\mathrm{P}=0.3)$.

\section{Cannabis use and persistence of psychotic experiences}

Analyses showed a significant association between continuation of cannabis use and risk of persistence of psychotic experiences (adjusted $\chi^{2} 16.22, \mathrm{P}=0.001$; tables 4 and 5). After adjustment for age, sex, socioeconomic status, use of other drugs at baseline and T2, urban/rural environment, childhood trauma, and occasional cannabis use (cannabis use at either baseline or T2, exposure states 1 and 2), effect sizes were attenuated, with significance only for the combination of cannabis use at both baseline and T2 (exposure state 3; adjusted odds ratio $2.2,1.2$ to $4.2 ; \mathrm{P}=0.016)$. Additional adjustment for any psychiatric diagnosis other than psychosis at baseline occasioned a slight reduction in the association between cannabis use at both baseline and T2 and the risk for persistence of psychotic symptoms $(2.0,1.0$ to $3.8 ; \mathrm{P}=0.037)$.

\section{Missing data}

Imputation of 20 datasets with the ICE routine in Stata 11.1, which imputes multiple alternatives based on available data, showed that the association between continued cannabis use and the risk of persistence of psychotic symptoms remained significant (adjusted $\chi^{2}$ 16.65; $\mathrm{P}=0.001$ ). Again, the strongest association was found for exposure state 3 (that is, cannabis use at both baseline and T2), with an adjusted odds ratio of 2.0 (1.1 to $3.7 ; \mathrm{P}=0.028)$.

\section{DISCUSSION}

This 10 year follow-up study showed that incident cannabis use significantly increased the risk of incident psychotic experiences. The association was independent of age, sex, socioeconomic status, use of other drugs, urban/rural environment, and childhood trauma; additional adjustment for other psychiatric diagnoses similarly did not change the results. There was no evidence for self medication effects as psychotic experiences did not predict later cannabis use. The results thus help to clarify the temporal association between cannabis use and psychotic experiences by systematically addressing the issue of reverse causality, given that the long follow-up period allowed exclusion of all individuals with pre-existing psychotic experiences or pre-existing cannabis use. In addition, cannabis use was confirmed as an environmental risk factor impacting on the risk of persistence of psychotic experiences (fig 3). 
Table $5 \mid$ Association between continued use of cannabis (over period from baseline to T2) and persistence* of psychotic experiences over period from T2 to T3. Figures are odds ratios ( $95 \%$ confidence intervals) and $P$ values

\begin{tabular}{lcc} 
Cannabis continuation & \multicolumn{2}{c}{ Risk of persistence of psychotic experiences } \\
\cline { 2 - 3 } No use & Unadjusted & Adjusted $†$ \\
\hline At baseline but not at T2 & 1 & 1 \\
\hline At T2 but not at baseline & $2.0(0.95$ to 4.4$), 0.068$ & 2.1 (0.9 to 4.7), 0.078 \\
\hline At baseline and T2 & $1.9(1.1$ to 3.2), 0.022 & $1.4(0.8$ to 2.5$), 0.202$ \\
\hline
\end{tabular}

*Persistence of psychotic experiences; present at T2 and T3.

†Adjusted for age, sex, socioeconomic status, use of other drugs baseline and T2, childhood trauma, and urban/rural environment. over time. ${ }^{35-38}$ In rats, repeated exposure to $\mathrm{THC}$ (delta-9-tetrahydrocannabinol, the main psychoactive component of cannabis) induces behavioural sensitisation: rats pre-treated with increasing doses of $\mathrm{THC}$ show greater behavioural (locomotor) responses to a THC challenge after a 14 day washout period than THC naive rats. ${ }^{3940}$ In humans, however, direct evidence for cannabis sensitisation is lacking. As our study showed that the risk of persistent psychotic experiences increases with longer periods of cannabis exposure, we suggest that a process of sensitisation underlies the association between cannabis and psychosis. $^{32}$

The mechanism behind the association

We investigated the association between cannabis use and risk of psychosis by analysing the expression of psychotic experiences. Psychotic experiences share many characteristics with clinically relevant psychosis, such as demographic, environmental, and genetic risks, and are thought to represent a behavioural marker for psychosis liability. ${ }^{183132}$ Psychotic experiences are a common and generally transitory phenomenon in the general population, that, nevertheless, might become abnormally persistent and progress to clinical psychotic disorder if combined with exposure to environmental risks. ${ }^{193334}$ Our study confirmed cannabis as an environmental risk factor, impacting on risk of psychosis by increasing the risk of incident psychotic experiences, and, if use continues over time, increasing the risk of persistent psychotic experiences.

The finding that longer exposure to cannabis was associated with greater risk for persistence of psychotic experiences is in line with an earlier study showing that continued cannabis use over time increases the risk for psychosis in a dose-response fashion. ${ }^{5}$ This is also in agreement with the hypothesis that a process of sensitisation might underlie emergence and persistence of psychotic experiences ${ }^{35}$ as an indicator of liability to psychotic disorder. ${ }^{1831}$ Sensitisation refers to the phenomenon that repeated exposure to an (environmental) stressor leads to progressively greater responses

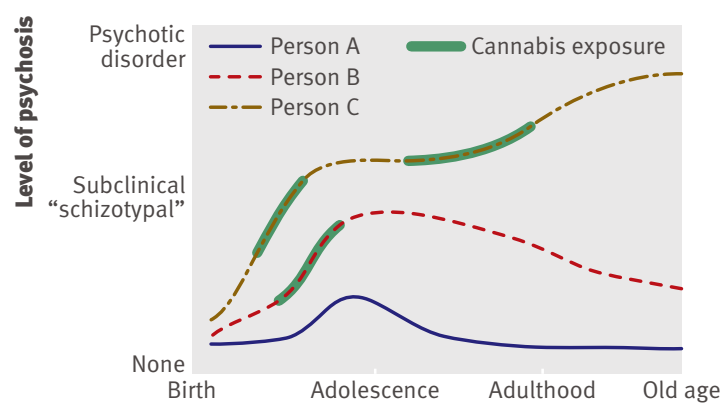

Fig 3 | Cannabis-psychosis persistence model. Person A has normal developmental expression of subthreshold psychotic experiences that are mild and transient. Person B has similar expression but longer persistence because of additional environmental exposure (here cannabis). Person $C$ has prolonged persistence and subsequent transition to clinical psychotic disorder because of repeated environmental exposure-that is, repeated cannabis use

\section{Methodological issues}

The results should be interpreted in the light of several limitations. Firstly, information on substance use and psychosis outcome was acquired with the DIA-X/MCIDI, which essentially provides self reported information. The interview was conducted face to face by clinical psychologists, however, who were allowed to follow up with clinical questioning to ensure systematic and valid assessment of outcomes and can therefore be assumed to yield better and more valid results than a self report questionnaire. Secondly, the analyses were not directly adjusted for the possible confounding effects of a family history of psychosis as this information was not available in the EDSP data. Previous research has shown that associations between cannabis use and psychotic symptoms are not reducible to family history of psychosis ${ }^{4142}$ and that genetic liability for psychotic disorder does not predict cannabis use. ${ }^{43}$ In addition, individuals with a family history of psychosis report more positive symptoms than individuals without such predisposition. ${ }^{445}$ As we excluded all individuals with at least one T2 lifetime psychotic symptom from the analysis, the possible confounding effect of family history for psychosis was indirectly adjusted for to a degree. Furthermore, we used a rather broad outcome measure, defined as a minimum of one positive rating on a $\mathrm{G}$ section item, representing psychotic experiences rather than clinically relevant psychotic disorder. It has been shown, however, that psychotic experiences show continuity with psychotic disorders such as schizophrenia. ${ }^{1846}$ In addition, given that fact transient psychotic experiences might, under certain circumstances, become abnormally persistent, giving rise to clinical psychotic disorder, ${ }^{15} 1719$ psychotic experiences represent an important phenotype for the investigation of mechanisms and pathways by which environmental risk factors such as cannabis impact on psychosis risk. A further limitation concerns the use of the G section of the DIA-X/M-CIDI. This rence of symptoms, which represents a long period for retrospective assessment of psychotic phenomena, possibly resulting in false negative results. As we excluded participants with T2 lifetime experience of psychotic symptoms from the analyses, under-reporting would have resulted in false negative results being incorrectly retained in the analyses. It is unlikely that section was administered at $\mathrm{T} 2$ to assess lifetime occur- 


\section{WHAT IS ALREADY KNOWN ON THIS TOPIC}

\section{Cannabis use is associated with increased risk for psychotic disorder}

The temporal association between cannabis use and the onset of psychotic symptoms, as well as the mechanism by which cannabis use leads to psychotic disorder, remain unknown

\section{WHAT THIS STUDY ADDS}

Cannabis use precedes the onset of psychotic symptoms in individuals with no history of psychotic experiences; incident cannabis use was associated with incident psychotic experiences four years later

Continued use of cannabis might increase the risk for psychotic disorder by impacting on persistence of (normally transitory) psychotic experiences in young people

under-reporting would have occurred as a function of cannabis use, which could have resulted in biased estimates. In addition, $23 \%$ of participants reported lifetime subclinical psychotic symptoms at T2, which is in keeping with the estimated $15-28 \%$ rate of subclinical psychotic symptoms in the general population. ${ }^{47}$ Therefore, the influence of under-reporting is probably limited. Finally, as the time between follow-up visits was four years on average, selective recall could have influenced the results. Spurious findings could have arisen if those with psychotic symptoms had better recall of earlier cannabis use. Given the well known link between psychosis liability and cognitive alterations, including impaired memory, any influence of selective recall would probably have been conservative rather than anti-conservative.

Contributors: $\mathrm{H}-\mathrm{UW}$ and RL were the principal investigators of the study. RK analysed the data in collaboration with $\mathrm{CH}$, JvO, and MH. RK drafted the paper. All authors contributed to subsequent drafts of the paper and the final version. JVO is guarantor.

Funding: The EDSP study is funded by grants of the German Ministry of Research, Education and Technology (01EB9405/6 and 01EB9901/6) and the Deutsche Forschungsgemeinschaft (DFG), and this paper is part of NIH grant R01DA016977-01, PL.

Competing interests: All authors have completed the Unified Competing Interest form at www.icmje.org/coi_disclosure.pdf (available on request from the corresponding author) and declare: no support from any organisation for the submitted work; no financial relationships with any organisation that might have an interest in the submitted work in the previous three years; and no other relationships or activities that could appear to have influenced the submitted work.

Ethical approval: The EDSP project was approved by the ethics committee of the Medical Faculty of the Technische Universitaet Dresden (No EK-13811).

Data sharing: No additional data available.

1 Wittchen HU, Behrendt S, Hofler M, Perkonigg A, Rehm J, Lieb R, et al. A typology of cannabis-related problems among individuals with repeated illegal drug use in the first three decades of life: evidence for heterogeneity and different treatment needs. Drug Alcohol Depend 2009;102:151-7.

2 Perkonigg A, Goodwin RD, Fiedler A, Behrendt S, Beesdo K, Lieb R, et al. The natural course of cannabis use, abuse and dependence during the first decades of life. Addiction 2008;103:439-51.

3 Wittchen HU, Frohlich C, Behrendt S, Gunther A, Rehm J, Zimmermann $P$, et al. Cannabis use and cannabis use disorders and their relationship to mental disorders: a 10-year prospectivelongitudinal community study in adolescents. Drug Alcohol Depend 2007;88(suppl 1):60-70S.

4 Moore TH, Zammit S, Lingford-Hughes A, Barnes TR, Jones PB, Burke $M$, et al. Cannabis use and risk of psychotic or affective mental health outcomes: a systematic review. Lancet 2007;370:319-28.

5 Van Os J, Bak M, Hanssen M, Bijl RV, de Graaf R, Verdoux H. Cannabis use and psychosis: a longitudinal population-based study. Am J Epidemiol 2002;156:319-27.
6 Henquet C, Krabbendam L, Spauwen J, Kaplan C, Lieb R, Wittchen $\mathrm{HU}$, et al. Prospective cohort study of cannabis use, predisposition for psychosis, and psychotic symptoms in young people. BMJ 2005;330:11

7 Arseneault L, Cannon M, Witton J, Murray RM. Causal association between cannabis and psychosis: examination of the evidence. $\mathrm{Br}$ Psychiatry 2004;184:110-7.

8 Semple DM, McIntosh AM, Lawrie SM. Cannabis as a risk factor for psychosis: systematic review. J Psychopharmacol 2005;19:187-94.

9 Degenhardt L, Hall W. Is cannabis use a contributory cause of psychosis? Can J Psychiatry 2006;51:556-65.

10 Degenhardt L, Hall W, Lynskey M. Testing hypotheses about the relationship between cannabis use and psychosis. Drug Alcoho Depend 2003;71:37-48.

11 Ferdinand RF, Sondeijker F, van der Ende J, Selten JP, Huizink A, Verhulst FC. Cannabis use predicts future psychotic symptoms, and vice versa. Addiction 2005;100:612-8.

12 Lieb R, Isensee B, von Sydow K, Wittchen HU. The early developmental stages of psychopathology study (EDSP): a methodological update. Eur Addict Res 2000;6:170-82.

13 Wittchen HU, Perkonigg A, Lachner G, Nelson CB. The early developmental stages of psychopathology study (EDSP): objectives and design. Eur Addict Res 1998;4:18-27.

14 McGrath J, Welham J, Scott J, Varghese D, Degenhardt L, Hayatbakhsh MR, et al. Association between cannabis use and psychosis-related outcomes using sibling pair analysis in a cohort of young adults. Arch Gen Psychiatry 2010;67:440-7.

15 Hanssen M, Bak M, Bijl R, Vollebergh W, van Os J. The incidence and outcome of subclinical psychotic experiences in the general population. Br J Clin Psychol 2005;44:181-91.

16 Spauwen J, Krabbendam L, Lieb R, Wittchen HU, van Os J. Evidence that the outcome of developmental expression of psychosis is worse for adolescents growing up in an urban environment. Psychol Med 2006;36:407-15.

17 Cougnard A, Marcelis M, Myin-Germeys I, De Graaf R, Vollebergh W, Krabbendam L, et al. Does normal developmental expression of psychosis combine with environmental risk to cause persistence of psychosis? A psychosis proneness-persistence model. Psychol Med 2007;37:513-27.

18 Van Os J, Linscott RJ, Myin-Germeys I, Delespaul P, Krabbendam L. A systematic review and meta-analysis of the psychosis continuum: evidence for a psychosis proneness-persistence-impairment model of psychotic disorder. Psychol Med 2009;39:179-95.

19 Dominguez MD, Wichers M, Lieb R, Wittchen HU, van Os J. Evidence that onset of clinical psychosis is an outcome of progressively more persistent subclinical psychotic experiences: an 8-year cohort study. Schizophr Bull 2011;37:84-93.

20 Zammit S, Allebeck P, Andreasson S, Lundberg I, Lewis G. Self reported cannabis use as a risk factor for schizophrenia in Swedish conscripts of 1969: historical cohort study. BM/ 2002:325:1199.

21 Grech A, van Os J, Jones PB, Lewis SW, Murray RM. Cannabis use and outcome of recent onset psychosis. Eur Psychiatry 2005;20:349-53.

22 Wittchen HU, Lachner G, Wunderlich U, Pfister H. Test-retest reliability of the computerized DSM-IV version of the Munich composite international diagnostic Interview (M-CIDI). Soc Psychiatry Psychiatr Epidemiol 1998;33:568-78.

23 Robins LN, Wing J, Wittchen HU, Helzer JE, Babor TF, Burke J, et al. The composite international diagnostic interview. An epidemiologic instrument suitable for use in conjunction with different diagnostic systems and in different cultures. Arch Gen Psychiatry 1988;45:1069-77.

24 Cottler LB, Helzer JE, Mager D, Spitznagel EL, Compton WM. Agreement between DSM-III and III-R substance use disorders. Drug Alcohol Depend 1991;29:17-25.

25 Wittchen HU, Robins LN, Cottler LB, Sartorius N, Burke JD, Regier D. Cross-cultural feasibility, reliability and sources of variance of the composite international diagnostic interview (CIDI). The multicentre WHO/ADAMHA field trials. Br J Psychiatry 1991;159:645-58.

26 Wittchen HU. Reliability and validity studies of the WHO-composite international diagnostic interview (CIDI): a critical review. J Psychiatr Res 1994;28:57-84.

27 Read J, van Os J, Morrison AP, Ross CA. Childhood trauma, psychosis and schizophrenia: a literature review with theoretical and clinical implications. Acta Psychiatr Scand 2005;112:330-50.

28 Krabbendam L, van Os J. Schizophrenia and urbanicity: a major environmental influence-conditional on genetic risk. Schizophr Bull 2005;31:795-9.

29 Van Os J, Krabbendam L, Myin-Germeys I, Delespaul P. The schizophrenia environment. Curr Opin Psychiatry 2005;18:141-5.

30 Fisher HL, Craig TK, Fearon P, Morgan K, Dazzan P, Lappin J, et al. Reliability and comparability of psychosis patients' retrospective reports of childhood abuse. Schizophr Bull 2009 October 7 [epub ahead of print].

31 Polanczyk G, Moffitt TE, Arseneault L, Cannon M, Ambler A, Keefe RS, et al. Etiological and clinical features of childhood psychotic 
symptoms: results from a birth cohort. Arch Gen Psychiatry 2010;67:328-38

32 Van Os J, Kenis G, Rutten B. The environment and schizophrenia. Nature 2010;468:203-12.

33 Rossler W, Riecher-Rossler A, Angst J, Murray R, Gamma A, Eich D, et al. Psychotic experiences in the general population: a twenty-year prospective community study. Schizophr Res 2007;92:1-14.

34 Van Rossum I, Dominguez MD, Lieb R, Wittchen HU, van Os J. Affective dysregulation and reality distortion: a 10-year prospective study of their association and clinical relevance. Schizophr Bull 2009 Sep 30 [epub ahead of print].

35 Collip D, Myin-Germeys I, Van Os J. Does the concept of sensitization provide a plausible mechanism for the putative link between the environment and schizophrenia? Schizophr Bull 2008;34:220-5.

36 Myin-Germeys I, Delespaul P, van Os J. Behavioural sensitization to daily life stress in psychosis. Psychol Med 2005;35:733-41.

37 O’Daly O, Guillin O, Tsapakis E, Martinez D, Shergill S, Murray RM. Schizophrenia and substance abuse co-morbidity: a role for dopamine sensitization? J Dual Diagn 2005;1:11-40.

38 Howes OD, McDonald C, Cannon M, Arseneault L, Boydell J, Murray RM. Pathways to schizophrenia: the impact of environmental factors. Int / Neuropsychopharmacol 2004;7(suppl 1):7-13S.

39 Cadoni C, Pisanu A, Solinas M, Acquas E, Di Chiara G. Behavioural sensitization after repeated exposure to delta 9 -

tetrahydrocannabinol and cross-sensitization with morphine. Psychopharmacology 2001;158:259-66.

40 Cadoni C, Valentini V, Di Chiara G. Behavioral sensitization to delta 9 tetrahydrocannabinol and cross-sensitization with morphine: differential changes in accumbal shell and core dopamine transmission. J Neurochem 2008;106:1586-93.

41 Andreasson S, Allebeck P, Engstrom A, Rydberg U. Cannabis and schizophrenia. A longitudinal study of Swedish conscripts. Lancet 1987;2:1483-6.

42 Van Os J, Bak M, Hanssen M, Bijl RV, de Graaf R, Verdoux H. Cannabis use and psychosis: a longitudinal population-based study. Am J Epidemiol 2002;156:319-27.

43 Genetic Risk and Outcome in Psychosis (GROUP) Investigators Evidence that familial liability for psychosis is expressed as differential sensitivity to cannabis: an analysis of patient-sibling and sibling-control pairs. Arch Gen Psychiatry 2010 Oct 4 [epub ahead of print].

44 Keshavan M, Montrose DM, Rajarethinam R, Diwadkar V, Prasad K, Sweeney JA. Psychopathology among offspring of parents with schizophrenia: relationship to premorbid impairments. Schizophr Res 2008;103:114-20.

45 Niemi LT, Suvisaari JM, Tuulio-Henriksson A, Lonnqvist JK. Childhood developmental abnormalities in schizophrenia: evidence from highrisk studies. Schizophr Res 2003;60:239-58.

46 Johns LC, van Os J. The continuity of psychotic experiences in the general population. Clin Psychol Rev 2001;21:1125-41.

47 Verdoux $\mathrm{H}$, van Os J. Psychotic symptoms in non-clinical population and the continuum of psychosis. Schizophr Res 2002;54:59-65.

Accepted: 31 December 2010 